\title{
Evidence Control System for the process University Degree accreditation
}

\section{Paúl Paguay-Soxo and Pamela Buñay}

Carrera de Ingeniería en Sistemas y Computación, Universidad Nacional de Chimborazo, Avda. Mariscal Sucre Km. 11/2 vía a Guano, Riobamba, Ecuador, 593

\section{Abstract}

The quality standards established by the higher education control agencies in Ecuador require the demonstration of the processes of Higher Education Institutions through the evidence of the activities, each period in accordance with the requirements of the regulatory entities. The present research presents the results of the implementation of a system of control of evidences in the Career of Engineering in Systems and Computing of the National University of Chimborazo as a tool for the generation

Corresponding Author: Paúl Paguay-Soxo

Received: 28 July 2017 Accepted: 5 September 2017 Published: 30 January 2018

Publishing services provided by Knowledge $\mathrm{E}$

(c) Paúl Paguay-Soxo and Pamela Buñay. This article is distributed under the terms of the Creative Commons

Attribution License, which permits unrestricted use and redistribution provided that the original author and source are credited.

Selection and Peer-review under the responsibility of the SIIPRIN Conference Committee.

\section{G OPEN ACCESS} and validation of the evidences. An analysis of the generic model of evaluation, diagnostic situation, development and implementation of the solution and analysis of the changes generated by the implementation was performed. The results evidenced that the time of search, validation and collection of evidence decreased, also the increase of the quality of the information according to established guidelines, on the other hand, the difficulties found in the execution of the project were unveiled.

Keywords: Computer System, Evidence, Higher Education, Software, Management.

\section{Introducción}

Según las conclusiones del evento TICAL 2015 [1]: "La gestión de las instituciones es también una actividad trascendental en la vida de las universidades. Son organizaciones como cualquier otra de servicios; quizás, en cierto sentido, más complejas y amplias. Una gestión eficiente y efectiva, en la actualidad, debe basarse en el uso inteligente de las TIC.", por otro lado Silvio [2] indica que "en la educación superior se pueden virtualizar cuatro espacios funcionales típicos de cada función de la Universidad: aulas virtuales, laboratorios virtuales, bibliotecas virtuales y oficinas virtuales.", esta última virtualización enfocada a los procesos administrativos como la recolección de evidencias con fines de acreditación. En todo esto las Tecnologías de la Información juegan un papel preponderante que ha cambiado los paradigmas a lo largo de la historia como la señala Mariño y otros [3], "El vertiginoso desarrollo de las 
tecnologías favoreció el paso de la sociedad industrial a la sociedad de la información y de ésta a la sociedad del conocimiento", a su vez la constante expansión de las TIC's en los procesos organizacionales han facilitado la incorporación de las mismas como lo señala Rodríguez y otros [4] "Las herramientas digitales han dejado de ser algo que se utilizaba cuando era posible acceder a un ordenador a algo que se ha convertido en cotidiano. Por tanto, las tecnologías digitales han pasado a convertirse en parte de nuestro entorno, casi omnipresentes, y, por tanto, integradas y no separadas de nuestra vida y de nuestros contextos y entornos de aprendizaje".

En la investigación de García [5], existen propuestas por parte de la Unión Europea de políticas para promover la Universidad Virtual, como es el caso del Espacio Europeo de Educación Superior (EEES), con lo cual se pretende conseguir el objetivo en el 2020, que entre sus metas está: "El establecimiento de un nuevo modelo de gestión, completamente soportado por tecnologías que permitan el control y la automatización de las tareas". Y concluye, "No obstante, la experiencia nos dice que pese a los momentos caóticos del comienzo de un cambio tecnológico, la madurez en dichos procesos termina por conseguirse."

En Ecuador, se pretende que la sociedad reciba una educación superior de calidad, al ser un servicio público debe ser llevado con ciertos parámetros que lo establece el organismo regulador llamado Consejo de Evaluación, Acreditación y Aseguramiento de la Calidad de la Educación Superior (CEAACES). Esto implica que la evaluación de la Educación Superior está relacionada con la calidad de los procesos que la constituyen, y de su aseguramiento. [6].

EL CEAACES entre sus parámetros e indicadores de evaluación establece la "Calidad de la información", la misma que evalúa la información y evidencias que la Unidad Académica proporciona de forma "puntual, pertinente, consistente, completa y formal" [7], que permite el análisis adecuado de la evaluación documental por parte del equipo técnico del CEAACES y el comité evaluador externo.

La evaluación de información requiere que se establezcan criterios, métodos para realizar la validación de información. Se requiere una planificación concreta para los criterios y la forma de cómo se llevará a la práctica. Los criterios se realizarán mediante la aplicación de parámetros e indicadores de evaluación; mientras los métodos a través de procedimientos concretos y la ayuda de los recursos necesarios para la realización positiva de los métodos para llevar a cabo el proceso de evaluación. [8]

En la Carrera de Ingeniería en Sistemas y Computación (CISYC) de la Universidad Nacional de Chimborazo (UNACH), el proceso de recolección, revisión y validación de evidencias se lo venía realizando de manera manual, archivando la información y sus 
evidencias en espacios amplios donde cada vez existe menos lugar para los documentos. Entre los problemas encontrados estaban: la búsqueda de información, para lo cual el responsable procedía a desplazarse hacia el archivo y realizar la búsqueda, incurriendo en el consumo de tiempo y esfuerzo. Otro problema era la asignación de revisores de la información, ya que al ser física la evidencia, esta se la movilizada a distintos responsables provocando en ciertas ocasiones pérdida de documentos o deterioro de los mismos, la constatación de entrega se lo manejaba a través de "checklists" en hojas de cálculo, creando varias versiones del mismo para las diferentes evidencias, lo que implicaba una demora en la generación de estos documentos, deficiencia en su validación y dificultad en la consolidación. Todo esto provoca una incidencia negativa en los procesos de acreditación de la carrera.

Como lo considera Díaz y otros [9] este tipo de escenarios dificulta gestionar ágilmente la información, por el tratamiento de grandes volúmenes de datos, lo que provoca pérdidas y errores por el empleo de métodos tradicionales, con el correspondiente consumo en materiales de oficina y excesivo esfuerzo por parte de las personas involucradas en este proceso.

Por tal motivo se necesita de sistemas informáticos que formen parte de los sistemas organizacionales que se integren con la información ya existente como es el caso del Sistema Académico Institucional (SICOA) y provean nueva información, que en este caso particular ayuden a la automatización de recolección y validación de evidencias.

Sin embargo ante esta situación deseada de gestión es importante resaltar lo mencionado por Guevara-Iñiguez y otros [10] "La gestión tiene un carácter axiológico, cultural y se desarrolla a través de métodos y procedimientos en los cuales se logra la implicación de todos los miembros y recursos de la comunidad universitaria con la intención de crearla."

Al final según como lo señala Botero [11] "Cada institución tendrá que hacer esfuerzos para construir su propio modelo, sin olvidar que actualmente existen otros referentes y experiencias". Para la presente investigación fue necesario el establecimiento de políticas por parte de las autoridades de la Carrera y Facultad para posibilitar la incorporación del mismo.

El presente trabajo muestra los resultados obtenidos de la implementación del Sistema Informativo de Control de Evidencias (SICEV) en la CISYC de la UNACH, la metodología aplicada en el ciclo de desarrollo, el proceso informativo establecido y los cambios originados tanto positivos como negativos en cuanto a los requerimientos con fines de acreditación. 
Al final se puede concluir que el papel de las Tecnologías de la Información en los procesos organizacionales en su gran mayoría ofrece mejoras a los mismos. La oportunidad de la información al contar con datos consolidados permiten mantener un monitoreo constante a las actividades de la Carrera y posibilita la toma de decisiones en los momentos adecuados, así como la posibilidad de incorporar nuevos procesos en la que no se requiera utilizar insumos y materiales como papel, impresiones, discos compactos, entre otros, que a su vez si se lleva a cabo de manera global, generaría un impacto a largo plazo en el ámbito medioambiental que en la actualidad tiene gran relevancia.

\section{Metodología de la Investigación}

Para el presente trabajo se utilizó un tipo de investigación cuasiexperimental, con el cual se ha provocado una situación (implementación del SICEV) para luego observar los cambios provocados en cuanto a la información de calidad requeridos por el CEAACES.

Así también se utilizaron métodos empíricos como:

- La entrevista, realizada a coordinadores de las diferentes comisiones de la carrera para el establecimiento de requerimientos del sistema y entrega de información.

- Consulta de documentos para establecer la línea base de las variables a analizar.

- Consulta bibliográfica con el cual se estudió el reglamento actual del CEAACES, el mismo que establece el proceso de evaluación del entorno de aprendizaje de carreras presenciales y semipresenciales de las Instituciones de Educación Superior (IES), así también artículos científicos indexados y bibliografía relacionada al tema.

- Observación de las variables previa y posterior a la implementación del SICEV.

- Criterio de Expertos, con el cual se obtuvo los aportes por parte de los encargados de la evaluación de evidencias.

Por otro lado se utilizó roles, artefactos y ceremonias de dos metodologías ágiles de desarrollo de software, estas fueron:

- Metodología SCRUM, de la cual se utilizaron los artefactos: historias de usuario, product backlog, sprint backlog, reuniones de planificación, reunión de verificación, reunión de retrospectiva y gráficos burndown charts para el monitoreo del proyecto. 
- Metodología XP, con la que se estableció de igual manera iteraciones con fases que empataban con las ceremonias de la metodología anterior como fueron: Fase de exploración (Product Backlog), Fase de planificación (reunión de planificación), Fase de Iteraciones (Sprints), Fase de producción (reunión de verificación). Por otro lado se utilizó la programación en parejas.

\subsection{Población y muestra}

El presente trabajo tomó como línea base el periodo abril - agosto 2016 y realizó el experimento con el SICEV en los periodos octubre 2016 - febrero 2017 y abril 2017 agosto 2017, con la participación de 29 docentes en cada periodo que corresponden a la totalidad de docentes la carrera, cada uno tenía asignado actividades por realizar de acuerdo a su distributivo de trabajo las mismas que debían ser constatadas con sus respectivas evidencias.

\subsection{Procedimiento para el establecimiento de requerimientos del sistema informativo.}

Para la creación del sistema se estableció reuniones de trabajo con las coordinaciones de la Unidad Académica como fueron: Comisión de carrera, Comisión de Evaluación Acreditación, Comisión de Normas ISO y Dirección de Carrera.

De las reuniones mantenidas, la Comisión de Evaluación - Acreditación establecía los lineamientos y requerimientos del sistema, los mismos que deben estar articulados a cada uno de los indicadores del reglamento de evaluación del CEAACES, a su vez se establecieron los problemas del sistema vigente a ser reemplazado, en la que los documentos físicos presentaban inconvenientes tanto para su recolección, almacenamiento y búsqueda posterior, sin olvidar la incidencia en el costo que representa tanto para el docente y Universidad la adquisición de los materiales e insumos.

En una segunda instancia el resto de comisiones, luego de conocer los requerimientos de la comisión de evaluación y acreditación definieron sus formatos y responsables de su grupo de trabajo para la recolección y entrega de las evidencias establecidas para el período académico. 


\subsection{Requisitos del sistema informático}

La CISYC de la UNACH haciendo frente a la problemática presentada, a través de la comisión de Carrera se planteó establecer un Sistema Informativo de Control de Evidencias, que entre sus componentes contará con un Sistema Informático, el mismo que permite:

- Subir las evidencias de los diferentes procesos establecidos por las comisiones de la carrera.

- Revisar y evaluar las evidencias de las actividades registradas en el sistema por parte de un revisor asignado.

- Emitir informes de las actividades que pueden ser: tareas aprobadas, no aprobadas, sin revisión, sin realizar, así como el porcentaje de cumplimiento y el detalle de cada una de las evidencias ingresadas.

- Ingresar/eliminar tipos de actividades, actividades, revisores, asignar revisores, roles y permisos.

- Registro de roles de usuario y permisos.

- Migración de información sobre docentes, de la carrera en el periodo vigente.

- Autenticación de usuarios mediante el Sistema Académico Institucional (SICOA)

- Notificaciones vía email sobre actividades pendientes tanto a docentes como a revisores.

\subsection{Procedimiento de registro y revisión de evidencias}

Paso 1: Las comisiones de la carrera realizaron una reunión estableciendo los entregables y responsables para el período académico.

Con los formatos, entregables y responsables de revisión establecidos se procedió a calendarizar los entregables junto con su revisor y fechas de entrega, siendo este cronograma aprobado por la dirección de carrera.

Paso 2: El cronograma de entregas es suministrado a la comisión de evaluación acreditación quien es la encargada del ingreso en el sistema informático denominado Sistema de Control de Evidencias - SICEV, creado para este fin, registrando las tareas junto con el revisor, y fechas respectivas. Una vez completada la carga de información, a través del sistema informático se notifica tanto a docentes y revisores la cantidad de entregables y revisiones a su cargo respectivamente, este proceso es de la misma automatizado en el sistema. 
Paso 3: Los docentes ingresarán al SICEV en las fechas establecidas donde deberán ingresar la información durante todo el semestre, a través de una interfaz web, el docente constantemente conoce el estado de sus tareas y si estas han sido o no aprobadas por el revisor encargado, cada tarea aprobada contribuye a la calificación final de cumplimiento de actividades.

Paso 4: La comisión de evaluación - acreditación en conjunto con el director de carrera establecen la fecha límite de subida de información, luego de la cual a través del SICEV se notificará a los docentes y revisores sus tareas y revisiones pendientes.

Paso 5: A partir de la notificación se estableció un tiempo de dos semanas para la verificación de tareas por parte de los revisores asignados y corrección de actividades no aprobadas. En caso de que un revisor no apruebe la actividad realizada, deberá especificar claramente el error o incumplimiento, el mismo que será notificado vía correo electrónico automáticamente, el docente deberá posteriormente corregir el documento y volver a subir, luego del cual el sistema enviará de la misma manera un correo electrónico especificando la actividad pendiente de revisión.

Paso 6: Al cumplir con las dos semanas de revisión y corrección de tareas, el sistema genera el reporte de cumplimiento de cada docente, el mismo que servirá de insumo para la evaluación final realizada por el director de carrera (coevaluación) como parte de la evaluación docente.

\subsection{Establecimiento de evidencias}

En los diferentes períodos académicos se solicita evidencias que deben ser presentadas por los docentes. Tomando como referencia el período abril - agosto 2016, las evidencias solicitadas fueron:

- Hoja de vida en formato físico y digital

- Portafolios docente y estudiantil

- Planes de clase

- Informe de rendimiento académico semestral

- Acuerdos y compromisos

- Tutorías

- Reactivos

- Matriz de Seguimiento al Desarrollo Académico

- Matriz de Investigación Formativa 
Los períodos octubre 2016 - febrero 2017 y abril 2017 - agosto 2017 las evidencias establecidas fueron:

- Investigación Formativa

- Informe de giras

- Ejecución de Proyecto Integrador

- Elección de directiva

- Acuerdos y compromisos

- Producto Desarrollado

- Informes de comisión de Seguimiento a graduados (Todos los meses)

- Informe de Investigación (Todos los meses)

- Informes de Comisión de Evaluación y Acreditación (Todos los meses)

- Informes de comisión de Carrera (Todos los meses)

- Informes de ISO (Todos los meses)

- Informes de Comisión de Vinculación (Todos los meses)

- Informe de comisión de prácticas pre profesionales (Todos los meses)

- Informe de comisión de titulación especial (Todos los meses)

- Informe de concursos con estudiantes

- Copia de contratos o acciones de personal. Dos últimos períodos

- Copia de certificados de capacitación 2016 - 2017

- Planificación práctica de laboratorio

- Cumplimiento de prácticas de laboratorio

- Seguimiento al sílabo

- Matriz de curso de capacitaciones 2016 - 2017

\subsection{Criterios de evaluación de la calidad de la evidencia}

De acuerdo a las exigencias del CEAACES, la información y evidencias que la Unidad Académica deberá ser "puntual, pertinente, consistente, completa y formal" [13], los cuales han sido establecidos para la presente investigación y que se observará su cambio posterior a la implementación del sistema informático SICEV, a continuación se especifica cada uno: 
- Legibles: La evidencia puede ser leída en su totalidad.

- Información pertinente: La evidencia tiene correspondencia con la actividad encomendada.

- Puntual: La evidencia ha sido entregada en los plazos establecidos.

- Consistente: Cumplimiento con los formatos establecidos por las diferentes comisiones de la carrera

- Formal: Evidencia contenga firmas y sellos requeridos de acuerdo al formato establecido.

- Completa: La evidencia tiene toda la información solicitada (Ejemplo: Los informes de tutoría se encuentran registradas de todos los meses)

\subsection{Código de colores de actividades}

Luego de los requerimientos establecidos se definió un sistema de evaluación de actividades a través del siguiente código de colores:

- Verde: Actividad realizada y aprobada

- Amarilla: Actividad realizada sin revisión

- Naranja: Actividad realizada y No aprobada

- Rojo: Actividad no realizada

\subsection{Fórmula de porcentaje de cumplimiento de actividades}

El monitoreo y evaluación de actividades siempre será importante para la toma de decisiones oportunas para lo cual, la calificación final del docente por parte del director de carrera en cuanto al porcentaje de cumplimiento de actividades, se estableció a través de la siguiente fórmula:

$$
P C D=\frac{T R A}{T T} * 100
$$

Dónde:

PCD = Porcentaje de Cumplimiento Docente

TRA = Número de Tareas Realizadas y Aprobadas

TT = Total de Tareas Designadas al Docente 


\subsection{Especificaciones técnicas del sistema informático}

El sistema fue desarrollado con herramientas de software libre las cuales se detallan a continuación:

- Plataforma Java JDK 1.8

- Servidor Web Apache 2.2

- Contenedor web Tomcat 8

- Base de datos Postgresql 9.4

- Sistema Operativo Centos 6.8

- Librerías de Componentes JSF 2.2 con Primefaces 6.0

- En cuento a la arquitectura se compone de varias capas basado en el paradigma Modelo Vista Controlador (MVC): acceso a datos, reglas de negocio, control, vistas.

- Para las cuentas de usuario, se estableció una conexión con el sistema académico institucional mediante servicios web SOAP, para la autenticación de docentes, con lo cual se utiliza una sola clave de acceso, para ambos sistemas.

\section{Resultados}

Los resultados obtenidos con la implementación del sistema son los siguientes:

Debido a la facilidad de recolección y validación, el número de evidencias solicitadas se amplió de 9 a 21 para los dos períodos octubre 2016 - febrero 2017 y abril 2017 agosto 2017 , representando un incremento de $57,14 \%$ con respecto al período abril agosto 2016.

Para la evaluación del período abril - agosto 2016 se realizó el checklist de las nueve evidencias, la evaluación fue de forma y no de fondo. El informe de cumplimiento lo realizó el docente coordinador de la comisión ISO 9001- 2009 de la carrera utilizando para este cometido tres semanas, mientras que para los dos períodos octubre 2016 febrero 2017 y abril 2017 - agosto 2017 el proceso de evaluación se realizó con una distribución de actividades a cinco docentes y tomando en cuenta los criterios de información de calidad establecidos en el apartado anterior, ocupando para la revisión una semana, reduciendo el tiempo a un $66,66 \%$. 
El porcentaje de cumplimiento en el período abril - agosto 2016 según los informes realizados fue del 53,01\% mientras que en octubre 2016 - febrero 2017 y abril 2017 agosto 2017 fue del 90,63\%.

Entre las ventajas encontradas luego de la implementación del SICEV se detallan las siguientes:

- Creación de tipos de actividades y actividades estandarizadas

- Registro de evidencias a través de un sistema informático 24/7.

- Informes para la toma de decisiones

- Porcentajes de cumplimiento por docente y carrera

- Acceso a las evidencias en todo momento

- Evidencias fáciles de encontrar

- Asignación de actividades a docentes de forma ágil.

- Revisión de evidencias más ágil, realizado por las diferentes comisiones

- Notificar a los docentes las tareas no realizadas mediante correo electrónico

A continuación se presentan algunas interfaces del sistema informático:

Reporte General de actividades: permite visualizar a todos los docentes con el número de tareas aprobadas, no aprobadas, sin revisión, sin realizar, el porcentaje de cumplimiento y detalle cómo se puede visualizar en la Fig. 1.

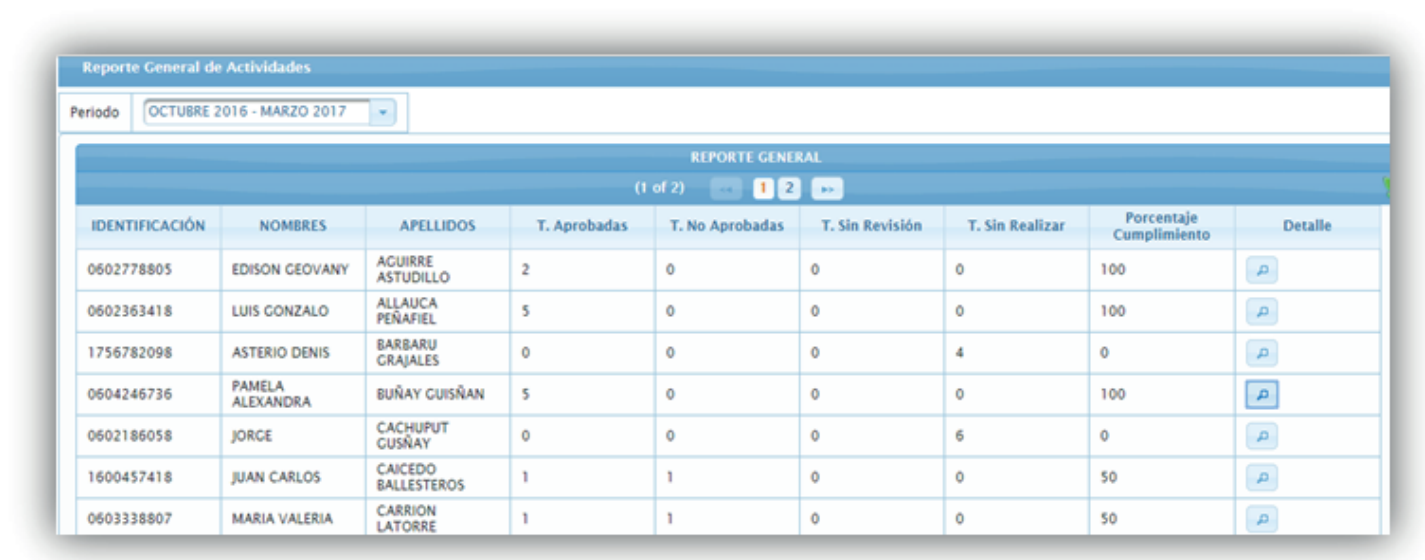

Figura 1: Reporte General de Actividades.

Reporte General de Actividades detallado: Permite visualizar las tareas de los docentes con más detalle y el porcentaje de cumplimiento como se puede visualizar en la Fig. 2 


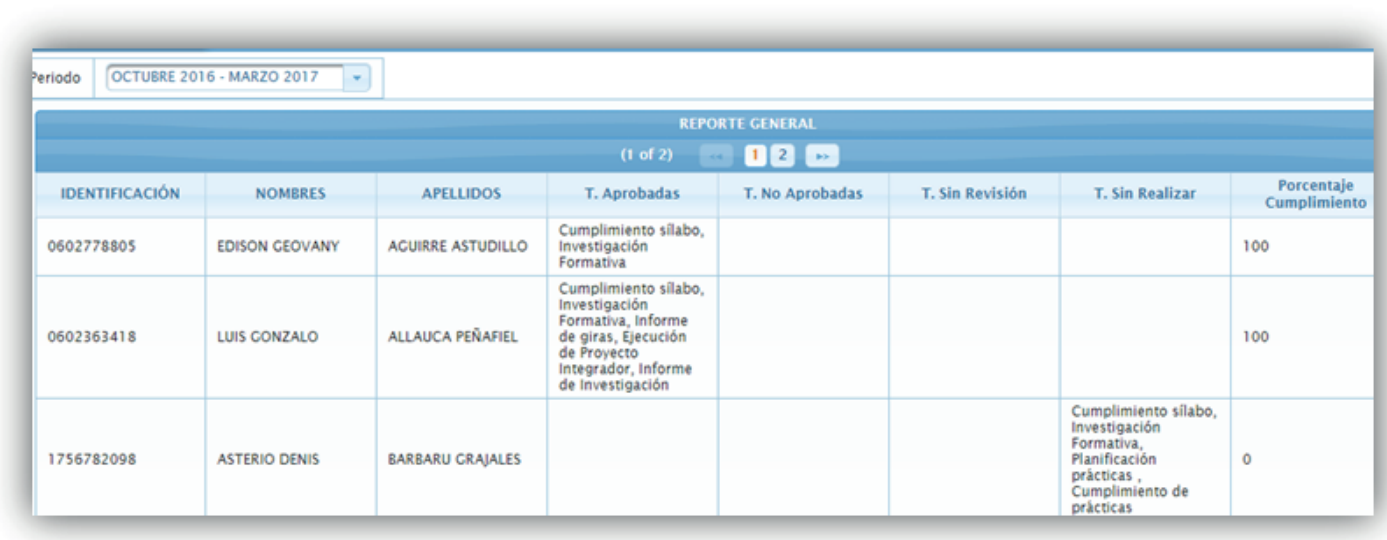

Figura 2: Reporte General de Actividades detallado.

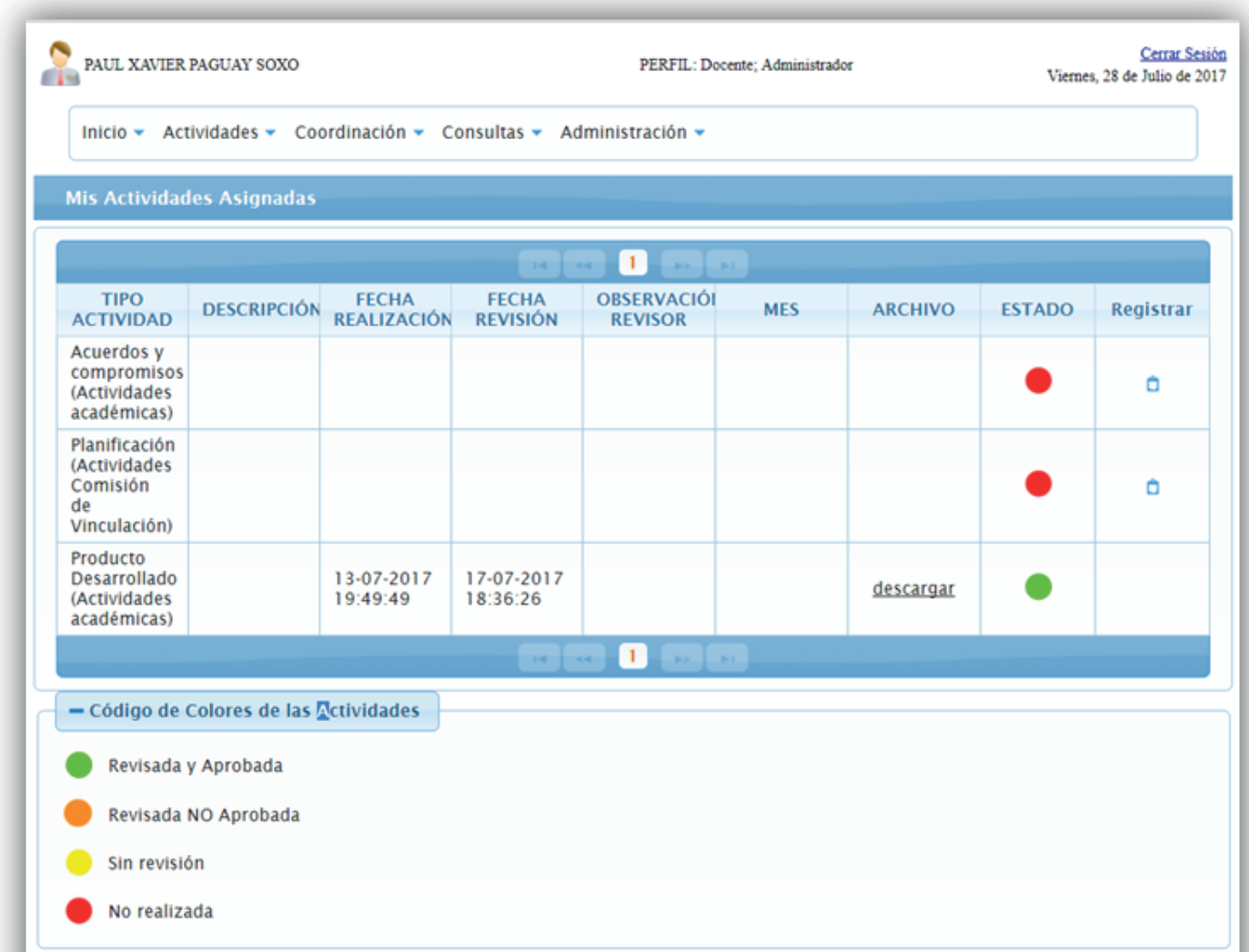

Figura 3: Registro de actividades del docente.

Visualización de actividades por parte del docente con su estado y opción de descarga la Fig. 3.

Toda acción de gestión requiere de procesos de retroalimentación para monitorear los resultados. Por tal motivo el sistema permitió obtener información de calidad, llevar 
un monitoreo de los resultados obtenidos de las diferentes actividades asignadas a los docentes de la carrera de Ingeniería en Sistemas y Computación, además que ayude a la gestión y toma de decisiones por parte de las autoridades. [1]

El sistema ha permitido que la información siempre esté disponible, por lo tanto, cuando se requiera alguna evidencia de las diferentes actividades realizadas, ésta sea fácil de encontrar y que cumpla con los diferentes parámetros de evaluación que son requeridos para las evidencias.

Al cumplir todo el ciclo, desde subir una actividad o tarea hasta que la tarea es aprobada por un revisor asignado por el director de carrera, ha permitido que el trabajo de recolección de evidencias al estar automatizado sea más ágil y eficaz.

Por otro lado, entre los aspectos negativos, un $9.37 \%$ de los docentes no cumplió con el registro de evidencias en el sistema informático, y se pudo observar que correspondían a docentes "compartidos" que trabajaban en otras carreras, por lo cual no participaban de las reuniones en las que se estableció y explicó el proceso.

\section{Conclusiones}

- Los sistemas informativos en los que intervienen sistemas informáticos, recurso humano e insumos, permiten establecer procedimientos normalizados, como es el caso del proceso de control de evidencias con fines de acreditación de la Carrera de Ingeniería en Sistemas y Computación, se han obtenido mejores resultados, en cuanto a la exigencia de calidad de información requerida por las entidades reguladora, así como el porcentaje de cumplimiento por parte de los docentes así también los tiempos de recolección, búsqueda y recuperación de información.

- Para la implementación de sistemas informáticos de gestión no solo en universidades sino en cualquier organización, es importante establecer las políticas y reglamentos para su utilización, con lo cual se promueve su uso y posterior establecimiento de procesos sostenibles en el tiempo.

- Es importante a futuro plantearse proyectos a nivel nacional en donde las universidades estandaricen los procesos de recolección de evidencias con políticas de cero papeles que no solo represente beneficios en cuanto a calidad de información o tiempos de respuesta como fue el caso del presente estudio, sino también beneficios económicos por el ahorro de materiales e insumos, a su vez con impacto positivo en el medioambiente. En este sentido, una opción muy concreta y realista es la creación de entidades certificadoras y firmas electrónicas. 


\section{Referencias}

[1] TICAL, ¿Las Tecnolog'ias de la Informaci'on y la Comunicacion potenciando la Universidad del Siglo XXI,i 2015.

[2] J. Silvio, ¿Universidades Virtuales en Iberoam'erica, ¿ Red Digital, 2001.

[3] S. Mari no, P. Alfonzo y M. Godoy, ¿Sistematizaci'on de trabajos de graduaci'on, una aproximaci'on a la gesti'on del conocimiento, ¿ textitEDUTEC, Revista Electr'onica de Tecnolog'ia Educativa, vol. 1, nं 57, pp. pp. 33-45, 2016.

[4] J. Rodr'iguez, C. Galv'an y F. Mart'inez, ¿El portafolio digital como herramienta para el desarrollo de competencias transversales, ¿ textitTESI. Teor'ia de la Educacion Educaci'on y Cultura en la Sociedad de la Informaci'on, vol. 14, ni 2, pp. pp. 157-177, 2013.

[5] F. J. Garc'ia Pe nalvo, ¿La Universidad de la pr'oxima d'ecada: La Universidad Digital, ¿ de textitProyecto de Excelencia de la Junta de Castilla y Le'on GR47, Salamanca Espa na, GRIAL, 2010, pp. pp 181-197.

[6] CEAACES, ¿MODELO PARA LA EVALUACIO N DE LAS CARRERAS PRESENCIALES Y SEMI-PRESENCIALES DE LAS UNIVERSIDADES Y ESCUELAS POLITECNICAS DEL ECUADOR, $\check{C}$ CEAACES, Quito, 2013.

[7] CEAACES, ¿MODELO PARA LA EVALUACIO N DE LAS CARRERAS PRESENCIALES Y SEMI-PRESENCIALES DE LAS UNIVERSIDADES Y ESCUELAS POLITECNICAS DEL

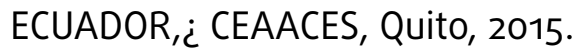

[8] J. A. M. Vega, ¿La evaluaci'on de la calidad de la informaci'on web: aportaciones, $i$ textitRecursos informativos: creaci'on, descripci'on y evaluaci'on. M'erida: Junta de Extremadura, p. 6, 2003.

[9] Y. D'iaz-Ricardo, Y. P'erez-del Cerro y D. Proenza-Pupo, ¿Sistema para la Gesti'on de la Informaci'on de Seguridad Inform'atica en la Universidad de Ciencias M'edicas del Holg'in, ¿ textitCiencias Holg'in, pp. pp. 1-14, 2014.

[10] E. Guevara-I niguez, M. E. Pardo y J. M. Izquierdo Lao, ¿Gesti'on acad'emica universitaria sustentada en las TICs. Caso Universidad Estatal, $¿$ textitRevista Pedag'ogica Maestro y Sociedad, vol. 12, ni 4, pp. pp. 31-39, 2015.

[11] C. Botero, ¿Cinco tendencias de la gesti'on educativa, $¿$ textitPOLIT'ECNICA, vol. 1, $n_{\dot{c}}$ 5, Pp. PP. 17-31, 2007.

[12] S. I. Mari no y M. V. Godoy, ¿Sistemas de gesti'on de informaci'on universitarios basados, ¿ textitRedUNCl, p. 10, 2003.

[13] H. L. Zamorano, ¿MODELOS DE SIMULACION PARA LA GESTION DEL CONOCIMIENTO Y SU MEDICI'ON, ¿ P. 12 
[14] D. Rodr'iguez G'omez, ¿Modelos para la creaci'on y gesti'on del conocimiento: una aproximaci'on te'orica, ¿ textitEDUCAR, p. 16, 2006.

[15] S. Gento, Instituciones educativas para la Calidad Total, Madrid: Madrid La Muralla, 1996.

[16] I. Gonz'alez, Calidad en la Universidad: evaluaci'on e indicadores, Salamanca: Universidad Salamanca, 2004.

[17] L. R. Gardu no, ¿Hacia un modelo de evaluaci'on de la calidad, ¿ textitRevista IBEOAMERICANA, p. 12, 199.

[18] S. Mari no y M. V. Godoy, ¿¿Un aporte hacia la Gesti'on del Conocimiento? Desarrollo de sistemas de informacion universitarios en la web, $\dot{c}$ de textitll Congreso Internacional de Sociedad de la Informaci'on y del Conocimiento, Madrid, Madrid : McGraw-Hill, Interamericana, 2003, pp. 233-238.

[19] V. A. Cabrera, ¿EL CONCEPTO CALIDAD EN LA EDUCACION UNIVERSITARIA: CLAVE PARA EL LOGRO DE LA COMPETITIVIDAD INSTITUCIONAL, ¿ textitRevista Iberoamericana de Educaci'on, vol. 35/5, p. 8, 2005.

[20] V. A. Cabrera, ¿EL CONCEPTO CALIDAD EN LA EDUCACION UNIVERSITARIA: CLAVE PARA EL LOGRO DE LA COMPETITIVIDAD INSTITUCIONAL, ¿ textitRevista Iberoamericana de Educaci'on (ISSN: 1681-5653), p. 8, 205.

[21] L. E. Gonz'alez, ¿“EL IMPACTO DEL PROCESO DE EVALUACION Y ACREDITACION EN LAS UNIVERSIDADES DE AMERICA LATINA", ¿ de textitLos Procesos de Acreditaci'on en el Desarrollo de las Universidades, Chile, Centro Interuniversitario de desarollo CINDA, 2005, P. 43.

[22] C. Torres y H. C'ardenas, ¿Sistema de indicadores acad'emicos para el monitoreo de un proceso de acreditaci'on en una Carrera de Ingenier'ia Industrial, ¿ textitUniversidad, Ciencia y Tecnolog'ia, vol. 17, ni 67, pp. pp. 123-133, 2013.

[23] M. Tamayo, El proceso de la Investigaci'on Cient'ifica, Ed. 4ta ed., M'exico D.F.: LIMUSA S.A., 2003, p. pp. 48. 